EDITORIAL

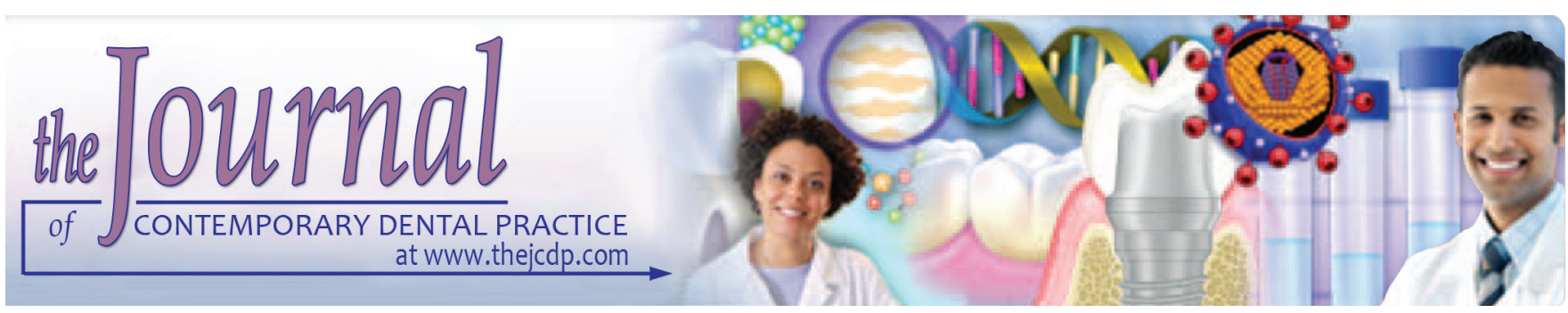

\title{
Tumor Budding in Oral Squamous Cell Carcinoma
}

\author{
${ }^{1}$ Gargi S Sarode, ${ }^{2}$ Sachin C Sarode, ${ }^{3}$ Revati Deshmukh, ${ }^{4}$ Rahul Anand, ${ }^{5}$ Shankargouda Patil
}

How to cite this article: Sarode GS, Sarode SC, Deshmukh R, Anand R, Patil S. Tumor Budding in Oral Squamous Cell Carcinoma. J Contemp Dent Pract 2017;18(9):743-744.

Source of support: Nil

Conflict of interest: None

\section{INTRODUCTION}

Tumor budding is defined as the presence of a single tumor cell or a small cluster of up to five cells in the tumor stroma, linking it to the epithelial-mesenchymal transition (EMT). ${ }^{1}$ Imai ${ }^{2}$ illustrated that tumors with this trait have more aggressive qualities and allocated the term "sprouting" instead of "budding." The term "tumor budding" was coined by Morodomi et al in 1989. ${ }^{3}$ Tumor budding in vitro or in experiments has been addressed by many names, depending on the researcher's background, few of which are tumor cell dissociation, focal dedifferentiation, or EMT. ${ }^{4}$

Tumor budding is the sequestration and deployment of cancer cells from a primary tumor through the initial stages of tumor invasion and is known to depict an effective cancerous invasion. Tumor cells undergoing EMT are an indication of tumor budding. Tumor budding can be considered as a histological prognostic marker as it predicts lymph node metastasis, lymphatic or vascular invasion, resistance to chemotherapy-radiotherapy, local recurrence, distant spread, and prognosis. Several human solid cancers associated with a poorer prognosis

${ }^{1-4}$ Department of Oral Pathology and Microbiology, Dr. D. Y. Patil Dental College \& Hospital, Dr. D. Y. Patil Vidyapeeth, Pune Maharashtra, India

${ }^{5}$ Department of Preventive Dental Sciences, College of Dentistry Jazan University, Jazan, Kingdom of Saudi Arabia

Corresponding Author: Gargi S Sarode, Department of Oral Pathology and Microbiology, Dr. D. Y. Patil Dental College \& Hospital, Dr. D. Y. Patil Vidyapeeth, Pune, Maharashtra, India show more areas of tumor budding as seen in esophageal squamous cell carcinoma (SCC), nasopharyngeal carcinoma, and laryngeal carcinoma. ${ }^{5}$ Tumor budding has also been detected in the tumor front in most of the oral squamous cell carcinomas (OSCCs), and a higher frequency of it recurring has been shown to be associated with an elevated number of budding. ${ }^{6}$

Interestingly, assessment of tumor budding can be done using hematoxylin and eosin-stained specimens, and thus can be easily done with routine histopathological examination. Moreover, the results achieved are reproducible. ${ }^{7}$ This assessment can be used as a significant prognostic marker and aid in a better treatment planning for the patient.

Tumor budding has received recent attention, particularly in the setting of colorectal carcinoma. Even though it is documented in several publications throughout the last few years, tumor budding as a histopathological predictive marker in head and neck SCC has been explored only recently.

\section{ORAL SQUAMOUS CELL CARCINOMA}

Oral cancer is among the top 10 cancers in the body. The survival of the patients has not enhanced significantly despite the progress in research and therapy. Thus, it represents a continuing challenge in the field of biomedical sciences and cancer researchers. ${ }^{8}$

The tumor microenvironment consists of cancerassociated fibroblasts (CAFs), immune cells, and other auxiliary cells. Changes in genetic profiles provide microenvironmental alterations, such as overproduction of cytokines, reactive oxygen species accretion, and EMT. ${ }^{8}$ Some markers for elements in OSCC tumor microenvironment include cytokeratin, E-cadherin, programmed death (PD)-ligand 1, FasL, CD33, CD144, cancer stem cell markers like aldehyde dehydrogenase, $\mathrm{N}$-cadherin, vimentin (EMT), integrin $\alpha 6$ (CAFs), CD4+CD25+FoxP3+ (T regulatory cells), CD8+, T-cell receptor, Fas, PD-1 (cytotoxic T cells), CD4+ (cytotoxic Th2 cells), CD34+ (myeloid precursor cells), etc. ${ }^{9}$ 


\section{TUMOR BUDDING IN OSCC: LESSONS LEARNED}

Marangon Junior et $\mathrm{al}^{10}$ found a convincing association between high level of tumor budding and amplified concentration of stromal alpha smooth muscle actin +ve myofibroblasts. Increased activity of tumor budding was found to be associated with high expression of laminin-5 gamma 2, suggesting that tumor budding plays a pivotal role in OSCC development.

Wang et $\mathrm{al}^{11}$ studied 230 patients with tongue SCC (TSCC) to examine tumor budding by investigating E-cadherin and vimentin expression. They detected tumor budding in 165 cases $(71.7 \%)$, with a mean tumor bud count of 7.5 ranging from 1 to 48 buds. Statistical analysis showed that tumor budding was linked with tumor size, clinical stage, differentiation, and lymph node metastasis and was concurrent with reduced survival. They also observed noteworthy correlation among tumor budding and deregulation of E-cadherin and vimentin. Hence, they concluded that tumor budding seems to be a self-determining prognostic factor in TSCC.

Xie et $\mathrm{al}^{12}$ retrospectively studied 195 patients with T1/T2 stage SCC of tongue. They reviewed tumor invasive depth, the concentration of tumor budding, and other clinicopathological characteristics and discovered that the incidence of tumor buds in tongue OSCC was about $85.6 \%$ in their study. They also noticed that the intensity of tumor budding showed strong correlations with occult lymph node metastasis, worse invasive pattern, invasive depth, and local relapse. They concluded that tumor budding which is a frequent event in TSCC can be used for routine pathological diagnosis and grading and while making the decision of elective lymph node dissection. It can also be applied independently to predict the prognosis of patients with T1/T2 stage TSCC.

Seki et $\mathrm{al}^{13}$ examined 91 cases of OSCC for conventional histopathologic assessment and tumor budding, and their association with lymph node metastasis. From the study they concluded that tumor budding should be added to routine histologic evaluation as a new measure of factoring into the decision as to whether neck dissection should be indicated or not.

\section{FUTURE DIRECTIONS}

The presence of tumor budding can be used as a promising prognosticator in OSCC. It can also be directed toward grading of OSCCs and help the surgeon to devise a better treatment plan regarding lymph node dissection.

\section{REFERENCES}

1. Koelzer VH, Zlobec I, Lugli A. Tumor budding in colorectal cancer-ready for diagnostic practice? Hum Pathol 2016 Jan;47(1):4-19.

2. Imai $\mathrm{T}$. The growth of human carcinoma: a morphological analysis. Fukuoka Igaku Zasshi. 1954;45:72-102.

3. Morodomi T, Isomoto H, Shirouzu K, Kakegawa K, Irie K, Morimatsu M. An index for estimating the probability of lymph node metastasis in rectal cancers. Lymph node metastasis and the histopathology of actively invasive regions of cancer. Cancer 1989 Feb;63(3):539-543.

4. Prall F. Tumourbudding in colorectal carcinoma. Histopathology 2007 Jan;50(1):151-162.

5. Okado Y, Aoki M, Hamasaki M, Koga K, Sueta T, Shiratsuchi H, Oda Y, Nakagawa T, Nabeshima K. Tumor budding and laminin5- $\gamma 2$ in squamous cell carcinoma of the external auditory canal are associated with shorter survival. Springerplus 2015 Dec;4:814.

6. Attramadal CG, Kumar S, Dhakal HP, Boysen ME, Nesland JM, Bryne M. P0094 Tumour budding in oral squamous cell carcinomas as a prognostic factor. Eur J Cancer 2015 Jul;51 (Suppl 2):e18-e19.

7. Hayes BD, Maguire A, Conlon N, Gibbons D, Wang LM, Sheahan K. Reproducibility of the rapid bud count method for assessment of tumor budding in stage II colorectal cancer. Am J Surg Pathol 2010 May;34(5):746-748.

8. Rivera C. Essentials of oral cancer. Int J Clin Exp Pathol 2015 Sep;8(9):11884-11894.

9. Curry JM, Sprandio J, Cognetti D, Luginbuhl A, Bar-ad V, Pribitkin E, Tuluc M. Tumor microenvironment in head and neck squamous cell carcinoma. Semin Oncol 2014 Apr;41(2):217-234.

10. Marangon Junior $\mathrm{H}$, Rocha VN, Leite CF, de Aguiar MC, Souza PE, Horta MC. Laminin 5 gamma 2 chain expression is associated with intensity of tumor budding and density of stromal myofibroblasts in oral squamous cell carcinoma. J Oral Pathol Med 2014 Mar;43(3):199-204.

11. Wang C, Huang H, Huang Z, Wang A, Chen X, Huang L, Zhou X, Liu X. Tumor budding correlates with poor prognosis and epithelial-mesenchymal transition in tongue squamous cell carcinoma. J Oral Pathol Med 2011 Aug;40(7):545-551.

12. Xie N, Wang C, Liu X, Li R, Hou J, Chen X, Huang H. Tumor budding correlates with occult cervical lymph node metastasis and poor prognosis in clinical early-stage tongue squamous cell carcinoma. J Oral Pathol Med 2015 Apr;44(4):266-272.

13. Seki M, Sano T, Yokoo S, Oyama T. Histologic assessment of tumor budding in preoperative biopsies to predict nodal metastasis in squamous cell carcinoma of the tongue and floor of the mouth. Head Neck 2016 Apr;38(Suppl 1): E1582-E1590. 